AperTO - Archivio Istituzionale Open Access dell'Università di Torino

\title{
ABVD vs BEACOPP escalated in advanced-stage Hodgkin's lymphoma: Results from a multicenter European study
}

\section{This is the author's manuscript}

Original Citation:

Availability:

This version is available http://hdl.handle.net/2318/1790895

since 2021-06-21T12:44:31Z

Published version:

DOI:10.1002/ajh.25871

Terms of use:

Open Access

Anyone can freely access the full text of works made available as "Open Access". Works made available under a Creative Commons license can be used according to the terms and conditions of said license. Use of all other works requires consent of the right holder (author or publisher) if not exempted from copyright protection by the applicable law. 


\section{American Journal of Hematology}

WILEY

\section{ABVD versus BEACOPP escalated in Advanced-Stage Hodgkin's Lymphoma: results from a multicenter European study}

\begin{tabular}{|c|c|}
\hline Journal: & American Journal of Hematology \\
\hline Manuscript ID & AJH-20-0207.R2 \\
\hline Wiley - Manuscript type: & Research Article \\
\hline $\begin{array}{r}\text { Date Submitted by the } \\
\text { Author: }\end{array}$ & $\mathrm{n} / \mathrm{a}$ \\
\hline Complete List of Authors: & $\begin{array}{l}\text { Mondello, Patrizia; Memorial Sloan Kettering Cancer Center } \\
\text { Caterina, Musolino; University of Messina, Internal Medicine - Division of } \\
\text { Hematology } \\
\text { Dogliotti, Irene; University of Turin } \\
\text { Bohn, Jan-Paul; Medical University of Innsbruck, Interval Medicine V } \\
\text { Cavallo, Federica; A.O.U. Città della Salute e della Scienza di Torino, } \\
\text { Clinica Ematologica } \\
\text { Ferrero, Simone; University of Torino, Department of Molecular } \\
\text { Biotechnologies and Health Sciences } \\
\text { Botto, Barbara; Azienda Ospedaliera Città della Salute e della Scienza, } \\
\text { Cerchione, Claudio; Universita degli Studi di Napoli Federico II } \\
\text { Nappi, Davide; Universita degli Studi di Napoli Federico II } \\
\text { De Lorenzo, Sonia; Hematology and T.M.O.-A.O.R.N. "S. G. Moscati", } \\
\text { Martinelli, Giovanni ; Istituto Scientifico Romagnolo per lo Studio e la } \\
\text { Cura dei Tumori } \\
\text { Wolf, Dominik; Medical University of Innsbruck, Internal Medicine V } \\
\text { Schmitt , Clemens ; Kepler University Hospital GmbH } \\
\text { Loseto, Giacomo; Hematology and Cell Therapy Unit - IRCCS Istituto } \\
\text { Tumori "Giovanni Paolo II", } \\
\text { Cuzzocrea, Salvatore; University of Messina, Biological and } \\
\text { Environmental Sciences } \\
\text { Willenbacher , Wolfgang ; Medical University of Innsbruck } \\
\text { Mian, Michael; Hospital of Bolzano, Hematology \& CBMT; Medical } \\
\text { University of Innsbruck, Department of Hematology \& Oncology } \\
\text { Straus, David; Memorial Sloan-Kettering Cancer Center, Medicine }\end{array}$ \\
\hline Keywords: & ABVD, BEACOPP, Hodgkin Lymphoma, advanced-stage, first-line therapy \\
\hline
\end{tabular}

\section{SCHOLARONE" Manuscripts}


Mondello et al, 2020

1

2

3 Patrizia Mondello MD, $\mathrm{PhD}, \mathrm{MSc}^{1,2}$, Caterina Musolino $\mathrm{MD}^{2}$, Irene Dogliotti $\mathrm{MD}^{3}$, Jan-Paul Bohn

$4 \mathrm{MD}^{4}$, Federica Cavallo $\mathrm{MD}^{3}$, Simone Ferrero $\mathrm{MD}, \mathrm{PhD}^{3}$, Barbara Botto $\mathrm{MD}^{5}$, Claudio Cerchione

$5 \mathrm{MD}, \mathrm{PhD}^{6}$, Davide Nappi $\mathrm{MD}^{7}$, Sonya De Lorenzo $\mathrm{MD}^{8}$, Giovanni Martinelli $\mathrm{MD}^{6}$, Dominik Wolf

$6 \mathrm{MD}^{4,9}$, Clemens Schmitt MD ${ }^{10}$, Giacomo Loseto MD ${ }^{11}$, Salvatore Cuzzocrea MD, $\mathrm{PhD}^{12}$,

7 Wolfgang Willenbacher $\mathrm{MD}^{4,13}$, Michael Mian $\mathrm{MD}^{14^{*}}$, David J Straus MD${ }^{1^{*}}$

8

$9{ }^{1}$ Department of Medicine, Memorial Sloan Kettering Cancer Center, New York, NY, USA

$10{ }^{2}$ Department of Human Pathology, University of Messina, Messina, Italy

11 3Hematology Division I, Department of Molecular Biotechnologies and Health Sciences,

12 University of Turin/AOU "Città della Salute e della Scienza di Torino", Turin, Italy

13 4Department of Internal Medicine V, Medical University of Innsbruck, Innsbruck, Austria

14 5Department of Hematology, "Città della Salute e della Scienza Torino", Turin, Italy

$15{ }^{6}$ Hematology Unit, Istituto Scientifico Romagnolo per lo Studio e la Cura dei Tumori (IRST)

16 IRCCS, Meldola, Italy

$17{ }^{7}$ Department of Medicine and Surgery, Hematology and Hematopoietic Stem Cell Transplant

18 Center, University of Naples Federico II, Naples, Italy

19 8Hematology and T.M.O.-A.O.R.N. “S. G. Moscati”, Avellino, Italy

209 Medical Clinic 3, University Hospital Bonn, Bonn, Germany

$21 \quad{ }^{10 K e p l e r ~ U n i v e r s i t y ~ H o s p i t a l ~ G m b H, ~ L i n z, ~ A u s t r i a ~}$

$22{ }^{11}$ Hematology and Cell Therapy Unit, IRCCS Cancer Institute "Giovanni Paolo II", Bari, Italy

$23{ }^{12}$ Department of Chemical, Biological, Pharmaceutical and Environmental Sciences, University

24 of Messina, Messina, Italy

$25{ }^{13}$ Oncotyrol, Center for Personalized Cancer Medicine, Innsbruck, Austria 


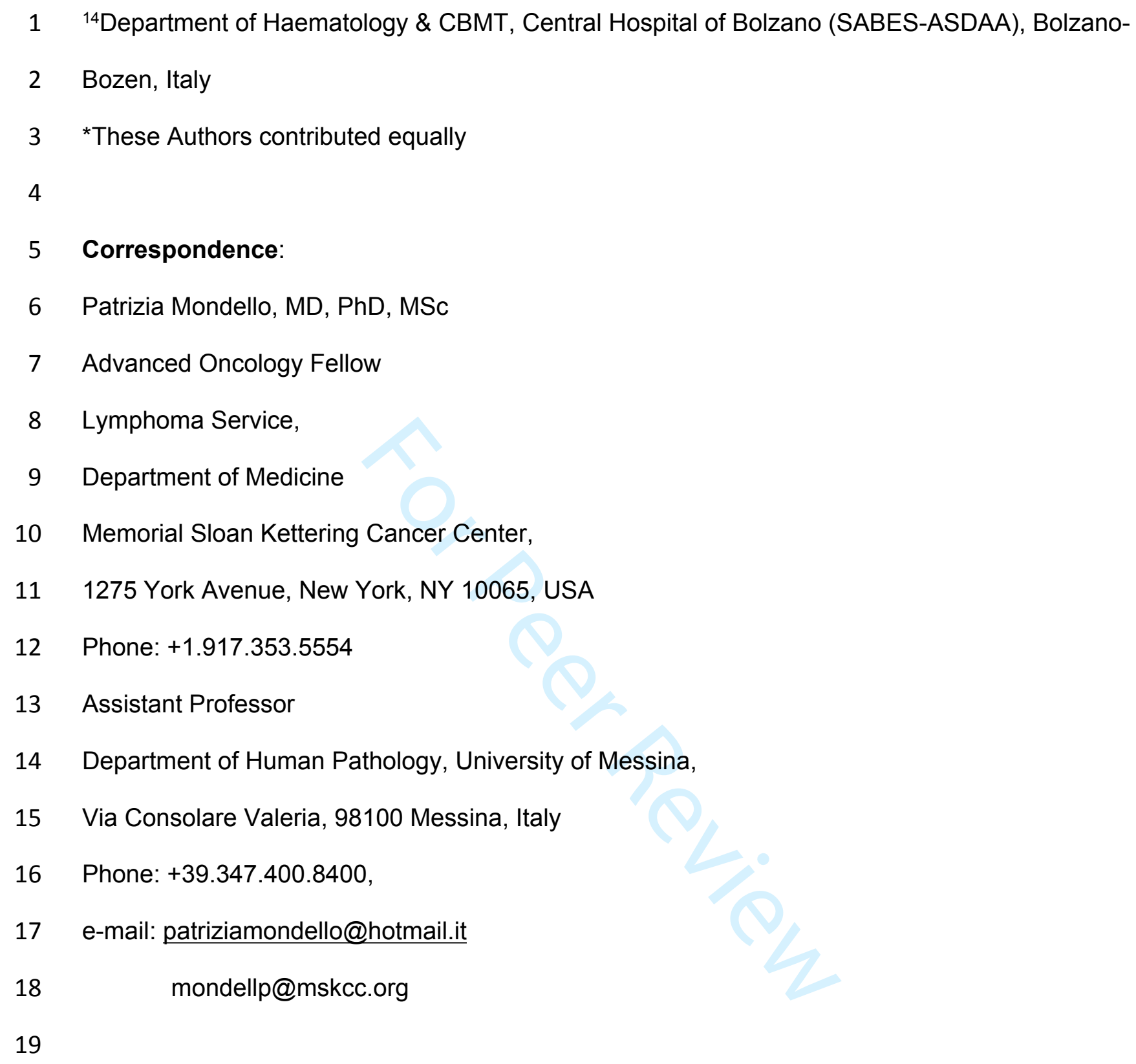




\section{References count: 35}

2

3 Key Points:

4 1. Although ABVD is associated with lower disease control than BEACOPP, it is better $5 \quad$ tolerated and leads to a similar overall survival rate.

62 2. BEACOPP is associated with severe, early and late toxicities, which may impact

7 survival.

8

9

10

11

12

13

14

15

16

17

18

19

20

21

22

23

24

25

26

John Wiley \& Sons 
Mondello et al, 2020

1

\section{Abstract}

3 The optimal first-line treatment for advanced-stage Hodgkin's lymphoma (HL) is still a matter of

4 debate. While ABVD is less toxic and as effective as other more intensive chemotherapy

5 regimens, escalated BEACOPP (BEACOPPesc) is superior to ABVD for initial disease control

6 and prolonged time-to-relapse. However, this advantage is associated with higher rate of early

7 and late toxicities. As most of these data have been accumulated from clinical trials, a

8 retrospective analysis was conducted in a large database of patients treated outside clinical

9 trials to investigate the advantages and disadvantages of these regimes in a real-world setting.

10 From October 2009 to October 2018, 397 advanced-stage HL patients treated with either ABVD

11 or BEACOPPesc were retrospectively assessed in 7 European cancer centers (2 Austrian and 5

12 Italian centers). Complete metabolic remission (CMR) by PET was achieved in $76 \%$ and $85 \%$ of

13 patients in the ABVD and BEACOPPesc groups, respectively ( $p=0.01)$. Severe adverse events

14 occurred more frequently with BEACOPPesc than ABVD. At a median follow-up of 8 years, $9 \%$

15 of the patients who achieved CMR after BEACOPPesc relapsed compared to $16.6 \%$ in the 16 ABVD group $(p=0.043)$. No statistical difference in progression free survival (PFS; $p=0.11)$ was

17 observed between the two cohorts overall, but there was a trend towards a superior PFS in 18 high-risk patients treated with BEACOPPesc $(p=0.074)$. Nevertheless, overall survival was 19 similar between the two groups $(p=0.94)$. In conclusion, we confirm that ABVD is an effective 20 and less toxic therapeutic option for advanced-stage HL. Although BEACOPP results in better 21 initial tumor control, the long-term outcome remains similar between the two regimens. 
Mondello et al, 2020

\section{Introduction}

Hodgkin's lymphoma $(H L)$ is a rare B-cell neoplasia accounting for about $11 \%$ of all newly diagnosed lymphomas in the Western World. ${ }^{1}$ This disease has a bimodal distribution with an increased incidence in young adults as well as in patients 55 years and older. ${ }^{2}$ The most commonly used first line treatment is ABVD (doxorubicin, bleomycin, vinblastine and dacarbazine). ${ }^{3}$ This regimen achieves remission in approximately $80 \%$ of patients with a relapse rate of about $25 \% .{ }^{4}$ Approximately $50 \%$ of those who progress or relapse after first line therapy can still be cured by salvage treatments including high dose chemotherapy (HDCT) followed by autologous bone marrow transplant (ASCT). ${ }^{4}$ In the attempt to improve cure rate with front line treatment, several groups developed novel combinations. The alternating combinations of ABVD and MOPP and more intensive chemotherapy regimens were explored; however, treatment results were similar and toxicity higher than with ABVD alone. ${ }^{5-7}$ Subsequently, the German Hodgkin Study Group (GHSG) developed BEACOPPesc (escalated bleomycin, etoposide, doxorubicin, cyclophosphamide, vincristine, procarbazine and prednisone), an intensified front line regimen, which demonstrated better initial disease control and prolonged time to relapse as compared with ABVD. ${ }^{8}$ However, this advantage was associated with higher rates of severe hematologic toxicity, treatment-related deaths, secondary neoplasms and infertility.9,10 Four clinical trials have directly compared ABVD and BEACOPP and all demonstrated a lower relapse rate after treatment with BEACOPP, but no difference in survival rates between the two regimens in primary data analysis..$^{9,11-13}$ Therefore, which regimen should be preferred as first line for advanced-stage HL has not been established yet.

Up to now, there has been no large real-world analysis comparing the efficacy and toxicity of these two regimens in advanced Hodgkin lymphoma outside of clinical trials. This is important because patients enrolled in clinical trials are often highly selected, and consequently may not reflect results in clinical practice. To shed some light on this open question we retrospectively assessed advanced $H L$ patients treated with either ABVD or BEACOPPesc in 
Mondello et al, 2020

1 seven European cancer centers outside of clinical trials and compared efficacy, safety and long-

2 term outcome of both regimens in a real-life setting.

3

42 Methods

$5 \quad 2.1$ Patients

6 From October 2009 to October 2018, treatment results in patients with newly diagnosed HL

7 stage III-IV who received either ABVD or BEACOPPesc outside of clinical trials were

8 retrospectively assessed from 7 European cancer centers: 2 Austrian and 5 Italian centers.

9 Histologic diagnosis was performed according to World Health Organization criteria by an expert

10 pathologist at each participating center. ${ }^{14}$ All patients were $\geq 18$ years or older. The clinical

11 stage of the disease was determined on the basis of a medical history and physical

12 examination; complete blood count; serum biochemical profile; chest radiography; computed

13 tomography of the chest, abdomen, and pelvis; positron-emission tomography (PET) with 18F-

14 fluorodeoxyglucose; and core biopsy of the iliac crest. To preserve fertility, ovarian stimulation

15 and cryopreservation of oocytes in females and sperm banking in males were performed before

16 the initiation of treatment. All patients were followed for disease progression/relapse, re-

17 treatment and death; all events were validated with medical records. The study was reviewed

18 and approved by the local ethics committees, and all patients provided written informed consent

19 before inclusion in the study. The study was conducted according to the Declaration of Helsinki.

\subsection{Treatment Plan}

22 First line treatment consisted of either six cycles of ABVD given every 4 weeks or six cycles of 23 BEACOPPesc given every three weeks, based on the physician's choice. For historical reasons, 24 the Austrian centers favored BEACOPPesc while the Italian ABVD. All patients underwent 25 routine inter-cycle hematological and biochemical evaluation. Starting within 1 month from the 26 end of chemotherapy, patients with residual metabolic active disease received high-energy 
Mondello et al, 2020

1 irradiation (30 Gy), with daily doses ranging between 1.5 and $1.8 \mathrm{~Gy}$. The Deauville 5-point

2 scoring system was used for assessment of treatment response. Complete metabolic response

3 (CMR), partial metabolic response (PMR), no metabolic response (NMR) and progressive

4 metabolic disease (PMD) determined by PET were defined according to international

5 lymphoma consensus guidelines. ${ }^{15,16}$ PET response was evaluated in all patients after two

6 cycles of treatment (PET2) and at the end of treatment. Patients with an interim PET showing

7 NMR or PMD received alternative treatment: those in the ABVD group received BEACOPPesc

8 while those in the BEACOPPesc group underwent further salvage treatment in accordance with

9 local protocols. After completion of the first line therapy, patients were evaluated every 3 months

10 during the first year, every 6 months from the second year through the fifth year after the

11 completion of treatment, and annually thereafter. All patients were evaluated for toxicity

12 according to the National Cancer Institute's Common Toxicity Criteria (NCI-CTC).

13 All patients who progressed or relapsed after a CMR were treated according to a salvage

14 chemotherapy program consisting of a reinduction regimen of multiple cycles of DHAP

15 (cisplatin, cytosine arabinoside and dexamethasone) or ifosfamide-containing therapy followed

16 by one high-dose course of autologous hematopoietic stem-cell-supported BEAM (carmustine,

17 etoposide, cytarabine and melphalan).

\section{$19 \quad 2.3$ Statistical Analysis}

20 The $\chi^{2}$ test was performed to assess the significance of differences between categorical 21 variables. PFS and OS were defined according to Cheson ${ }^{15}$ and plotted as curves using the 22 Kaplan-Meier method. Statistical analyses were performed with MedCalc (version 11.0; 23 MedCalc Software, Ostend, Belgium, http://www.medcalc.org) and GraphPad Prism (version 24 5.0; Graph-Pad, San Diego, CA, http://www.graphpad.com). All tests were two-sided. The limit 25 of significance for all analyses was defined as $p<0.05$. 
Mondello et al, 2020

1

\section{Results}

\section{3.1 Patient Characteristics at Start of Treatment}

4 A total of 397 consecutive patients were included in the study. One-hundred and twenty-one 5 patients were treated with BEACOPPesc and 276 with ABVD (Supplemental Table 1). The

6 baseline characteristics of the two groups did not differ significantly (Table 1). Overall, the 7 median age at time of diagnosis was 37 years (range, 19-75). A male predominance was 8 observed $(n=269,67.7 \%)$. Most patients had nodular sclerosing subtype $(n=302,76 \%), B$ 9 symptoms $(n=219,55.1 \%)$ and elevated erythrocyte sedimentation rate (ESR, $n=226,56.9 \%)$. 10 Only a minority had bulky disease $(\mathrm{n}=86,21.6 \%)$, defined as a tumor mass of $>7 \mathrm{~cm}^{17}$, and a 11 mediastinal mass $(n=66,16.6 \%)$. A lower proportion of patients with low international prognostic 12 score (IPS 0-1) received BEACOPPesc compared with ABVD (12.4\% vs 28.3\% respectively; $p=$ 13 0.041), while no differences were observed in the other IPS groups.

\subsection{Treatment Response}

16 After two cycles of treatment, a CMR was achieved in $69 \%$ of the ABVD group and in $78 \%$ of 17 the BEACOPPesc group $(p=0.003)$, respectively. Seventy-two $(25.6 \%)$ of the ABVD group 18 achieved a PMR, $4(1.4 \%)$ an NMR and $11(4 \%)$ had a PMD. In contrast, 17 of the patients in 19 the BEACOPPesc group had a PMR (14\%), 6 progressed $(5 \%)$ and another $4(3 \%)$ interrupted 20 therapy because of life-threatening toxicity. Patients with NMR or PMD at interim PET were 21 escalated to BEACOPPesc or received salvage treatment if they were previously treated with 22 ABVD $(n=15)$ or BEACOPPesc $(n=10)$, respectively. At the end of the therapy, CMR was $76 \%$ in 23 the ABVD group and $85 \%$ in the BEACOPPesc group $(p=0.01)$. A total of $20 \%$ of patients in the 24 ABVD group and $14 \%$ of patients in the BEACOPPesc group received consolidation involved 25 field radiotherapy to residual metabolic active disease at the dose of $30 \mathrm{~Gy}$. After radiotherapy, 26 the number of patients with CMR increased to $81 \%$ and $90 \%$ in the two groups, respectively $(p=$ 
1 0.021). All patients that failed to respond or progressed at end of treatment PET received a

2 salvage regimen followed by HDCT with autologous hematopoietic stem-cell support.

3 Forty-two patients (35\%) in the BEACOPPesc group required chemotherapy dose reduction due

4 to toxicity compared to 14 patients $(5 \% ; p=<0.001)$ in the ABVD group. Overall, the rate of

5 severe toxicities was higher in the BEACOPPesc group in comparison with the ABVD cohort.

6 There was a significant increased frequency of acute grade 3-4 hematologic adverse events in

7 the BEACOPPesc group as compared with the group treated with ABVD (neutropenia $61 \%$ vs

$824 \%, p<0.001$; anemia $29 \%$ vs $4 \%, p<0.001$; thrombocytopenia $29 \%$ vs $3 \%, p<0.001$ ), febrile

9 neutropenia $(29 \%$ vs $3 \%, p<0.001)$ and severe infections $(18 \%$ vs $3 \%, p=0.002)$. Myeloid

10 growth factors were administered to $85 \%$ of patients in the BEACOPPesc group compared with

$1159 \%$ in the ABVD group $(p=0.01)$. Figure 1 Blood transfusions were required in $41 \%$ of patients

12 in the BEACOPPesc group compared with $6 \%$ in the ABVD cohort $(p=<0.001)$. No case of

13 death due to acute treatment-related toxicity was registered in either group.

153.3 Follow-up

16 Progression during or shortly after treatment occurred in 6 patients in the BEACOPPesc group $17(4.9 \%)$ and in 16 patients in the ABVD group $(5.8 \% ; p=0.71)$. During the median follow-up 18 period of 8.7 years (range, 6.5-10.6 years), 10 out of 109 patients who achieved a CMR after 19 BEACOPPesc relapsed (9\%) compared to 37 of 223 patients in the ABVD group (16.6\%; $p=$ 20 0.043). However, no statistical difference in 8-year PFS rate was observed between the 21 BEACOPPesc and ABVD cohorts (Figure 2A, 80\% vs 75\%; $p=0.11$ ) irrespective of PET2 status 22 (Supplemental Figure 1A-B, 84\% vs 80\%; p=0.42 in PET2-negative patients and 69\% vs $2363.5 \% ; p=0.12$ in PET2-positive patients treated with BEACOPPesc and ABVD, respectively).

24 The baseline international prognostic score (IPS $<3$ vs $\geq 3)^{18}$ predicted a trend towards higher 25 PFS for the high-risk group treated with BEACOPPesc as compared with ABVD (Supplemental 26 Figure $2 \mathrm{~A}-\mathrm{B}, 81 \%$ vs $72 \%$; $\mathrm{p}=0.074)$. However, there was no difference in 8 -year OS rate 
Mondello et al, 2020

1 between the two treatment groups (Figure 2B; $90 \%$ vs $87 \%$; $p=0.94$ ) irrespective of PET2

2 status (Supplemental Figure 1C-D, 93.7\% vs 91\%; $p=0.86$ in PET2-negative patients and $77 \%$

3 vs 78.8\%; $p=0.61$ in PET2-positive patients treated with BEACOPPesc and ABVD, respectively)

4 or IPS risk score (Supplemental Figure 2C-D, $85 \%$ vs $83 \%$; $p=0.81$ in IPS $<3$ patients and

$577 \%$ vs $79 \% ; p=0.94$ in IPS $\geq 3$ patients in the BEACOPPesc and ABVD group, respectively).

6 During the follow-up period, secondary malignancies were observed in $5.8 \%$ of the patients

7 treated with BEACOPPesc compared to less than $1 \%$ of those who received ABVD. The median

8 time from the end of treatment to diagnosis of second malignancy was 80 months (range, 3-115

9 months). All patients treated with BEACOPPesc who developed second malignancies were in

10 first remission, while the ones in the ABVD cohort received second line therapy followed by

11 autologous transplant. In particular, three patients in the BEACOPPesc group developed

12 myelodysplasia and one acute leukemia. Second solid tumors developed in two patients in the

13 ABVD group (one lung cancer and one breast cancer) and seven in the BEACOPPesc group

14 (four breast cancer, one lung cancer, one melanoma and one thyroid cancer). Table 2 Four of

15 these 9 patients received radiotherapy as part of their initial treatment, with tumor development

16 within or close to the irradiated field (one lung and one breast cancer in ABVD group, and one

17 lung and one thyroid cancer in the BEACOPPesc cohort).

\section{Discussion}

20 The preferred treatment for advanced Hodgkin's lymphoma has been a matter of discussion for

21 three decades. Four international clinical trials addressed this important question by directly 22 comparing ABVD and BEACOPPesc. ${ }^{9,11-13}$ All of them demonstrated the superiority of 23 BEACOPP in achieving disease control; however, this benefit did not translate into long term 24 advantage due to a higher rate of late major events, particularly second malignancies, which led 25 to treatment-related patient deaths and ultimately comparable survivals to ABVD.9,10,12,13 Hence, 26 a balance between efficacy and toxicity should be considered in selecting the first line treatment 
Mondello et al, 2020

1 for these patients. Since a real-world analysis was lacking, we retrospectively assessed a large

2 European cohort of advanced stage $\mathrm{HL}$ patients who underwent either ABVD or BEACOPP

3 outside of clinical trials. This study provides real-world data confirming that ABVD is better

4 tolerated and has similar survival rate as BEACOPPesc as previously reported in published 5 clinical trials.

6 The main limitations of this study were the retrospective rather than prospective analysis 7 and lack of central review of the diagnostic pathology and PET images. The strengths of this

8 analysis were the relative long follow-up and the uniform treatment in a multicenter setting 9 although patients were treated outside a clinical trial.

10 Patient characteristics at the time of diagnosis were comparable between both treatment 11 groups and similar to those of other published cohorts, $92,13,19$. As expected, a lower proportion of 12 patients with IPS $0-1$ received BEACOPPesc compared to ABVD (12.4\% vs $28.3 \%$, 13 respectively; $p=0.041$ ). For historical reasons, BEACOPP was the preferred first line regimen in

14 the Austrian centers compared to the Italian ones ( $80.2 \%$ vs $19.2 \%)$. This different therapeutic 15 preference among centers may actually be an advantage as a similar patient population was 16 treated with either regimen. In the present analysis, the response rate of patients who received 17 BEACOPPesc was superior to those who received ABVD ( $85 \%$ vs $76 \%, p=0.01$ ). Interestingly, 18 the rate of patients with disease progression and interruptions for life threatening toxicity was 19 high in the BEACOPPesc group (8\%) relative to $5.4 \%$ of NMR/PMD with ABVD. Our results are 20 in line with three prospective studies using the same treatment program, which obtained a 21 complete response rate between $85-96 \%$ and $73-85 \%$ in the BEACOPPesc and ABVD groups, 22 respectively. ${ }^{8,9,13}$ However, EORTC 20012 and HD2000 failed to show a difference in CR ${ }^{11,12}$ 23 between the two regimens probably due to the poor tolerance of the BEACOPPesc regimen 24 which ultimately led to dose reduction or treatment discontinuation. Indeed, the high activity of 25 BEACOPPesc is associated with severe acute hematologic and non-hematologic toxicities. In 26 our study $93 \%$ of patients who underwent this intensive treatment regimen experienced at least 
Mondello et al, 2020

1 one severe acute toxic effect, most commonly myelosuppression and febrile neutropenia. This

2 translated into superior costs for growth factor and antibiotic use, supportive measures and

3 hospitalization, and led to the permanent discontinuation in 4 cases. These results are

4 comparable to those of the four prospective trials where severe hematologic toxicity was also

5 the most frequent adverse event. $9,12,13,19$ Considering the young age of patients with $\mathrm{HL}$, even

6 more important is the long-term toxicity associated with BEACOPP, which ultimately affects

7 overall survival related to the higher incidence of second malignancies with BEACOPPesc

8 compared with ABVD. In our study the rate of secondary malignancy was $5.8 \%$ in the patients

9 treated with BEACOPPesc. Similarly, the GHSG H9, HD12 and HD2000 trials reported a

10 cumulative risk for second malignancies between $4.9 \%$ and $6.5 \% .8,10,20$

11 Previous studies showed a superior PFS at 5 years in patients treated with BEACOPP

12 compared to ABVD 11,13; however this advantage was lost with a longer observation time ${ }^{9,10}$.

13 Similarly, we did not observe difference in long-term outcomes between the two regimens

14 irrespective of the International Prognostic Score and PET2 status. One explanation may be the

15 high efficacy of salvage therapies after ABVD. Another explanation might be the higher

16 incidence of secondary malignancies in patients treated with BEACOPP, which is consistent

17 with previous reports ${ }^{10,12}$. It should also be noted that despite its historical importance, IPS has

18 lost its prognostic value in the modern era using PET-guided therapy. ${ }^{29}$ Nevertheless, it is also

19 possible that a larger study may have captured the difference between the two treatment

20 groups. In addition, our study used 6 cycles of BEACOPPesc while 4 cycles has become the

21 standard in PET2-negative patients since 2018. ${ }^{21}$ This PET-adapted treatment led to less

22 severe acute toxicities, and ultimately to a slightly superior 5-year OS compared with the

23 extended treatment ( $97.7 \%$ vs $95.4 \%)$; however, the incidence of second malignancies did not

24 differ between the two treatment groups $(3.3 \% \text { vs } 3.8 \% ; p=0.37)^{21}$, suggesting the need for a

25 longer observation period for occurrence of further second malignancies to confirm the small

26 survival advantage. It is known that the addition of radiotherapy to chemotherapy increases the 
1 risk of secondary malignancies. However, in our study a similar number of patients in either

2 treatment group received additional radiation therapy for residual disease. In contrast to our

3 data, the HL9 trial demonstrated a superior 10-year OS for BEACOPPesc compared with

4 COPP/ABVD, which has similar survival to $A B V D^{22}$. It should be noted that this was an

5 unplanned secondary late analysis and not the primary endpoint of the trial. It should also be

6 noted that survival has improved with ABVD in recent retrospective analyses outside of a clinical

7 trial setting..$^{23}$ Although a meta-analysis from the GHSG group comparing BEACOPP and

8 ABVD showed a $10 \%$ OS benefit favoring BEACOPP ${ }^{24}$, these results were reported after only 5

9 years of follow-up, and 10-year results are needed to observe long-term toxicities and

10 consequent loss of survival advantage.

11 Achieving high cure rates while lowering early and late treatment-related toxicity remains

12 a challenge for patients with advanced-stage Hodgkin's lymphoma. The use of functional

13 imaging with PET early in the course of therapy offers a way to make treatment adjustment

14 based on response to therapy. Recent studies have showed that excellent results can be

15 achieved by using PET2 to modulate therapy, with escalation for those with an unsatisfactory

16 response and de-escalation for those with chemosensitive disease. ${ }^{25-28}$ Such an approach

17 would spare the $70 \%$ of patients destined to be cured by ABVD from receiving the more toxic

18 BEACOPP regimen, and avoid prolonged exposure to BEACOPP to those who do not need it.

19 The RATHL (Response-Adapted Therapy in Advanced-Stage Hodgkin Lymphoma) trial showed

20 that in PET2-negative patients the bleomycin-deleted AVD was as good as ABVD, hence

21 reducing lung toxicity, with a 3 -year PFS of $86 \%$. In those who were PET2-positive after two

22 cycles of ABVD, switching to BEACOPPesc seemed to improve outcomes, with a 3-year PFS

23 rate of $71.1 \%$ and OS rate of $82.8 \%{ }^{25}$ In support of the predictive power of PET in this setting,

24 the Italian GITIL0607 study showed that PET2-negative patients treated with continued ABVD

25 regimen reached a 3-year PFS of $87 \%$. In this trial, the randomization of PET2-negative patients

26 to receive or not RT did not show a PFS advantage. ${ }^{30}$ However, in our study the outcome of 
Mondello et al, 2020

1 PET2-negative patients is more disappointing than the 2 larger studies with an 8-year PFS of

$284 \%$ and $80 \%(p=0.42)$ in the two treatment cohorts. Our data are in line with the SWOG0816

3 trial which reported a 5-year PFS of only $74 \%$ for PET2-negative patients who received 6 cycles

4 of ABVD. ${ }^{31}$ The longer follow-up of our analysis and of the SWOG trial might be explanatory of

5 the difference to the 2 previous studies. Indeed, toxicity related deaths occur more frequently

6 beyond 3 years after chemotherapy. To further argue against the predictive power of PET, the

7 HD18 trial showed that the two arms of patients with advanced Hodgkin lymphoma who were

8 PET2-positive after 2 cycles of BEACOPPesc and continued to a total of six/eight cycles

9 reported 5-year PFS rates of $89.7 \%$ and $88.1 \%$, respectively. No significant differences in PFS

$10(p=0.30)$ and OS $(p=0.49)$ were observed between PET2-positive and PET2-negative

11 patients. ${ }^{21}$

12 Several efforts have been made to incorporate novel molecules into the backbone of the

13 two first line regimens to improve efficacy of ABVD and decrease toxicity of BEACOPP.

14 Recently, Brentuximab vedotin (BV), an anti-CD30 antibody-drug conjugate, has shown

15 remarkable activity in Hodgkin lymphoma. ${ }^{32}$ In an attempt to improve efficacy of $A B V D$, the

16 ECHELON-1 phase III trial evaluated a modified variant which replaced bleomycin with BV (A-

17 AVD) in advanced Hodgkin lymphoma patients. The 2-year modified PFS rate favored A-AVD

$18(82.1 \%$ vs $77.2 \%, p=0.004),{ }^{33}$ leading to the FDA approval of this new combination. However,

19 the study has not yet showed a benefit in overall survival since the number of events has not

20 been reached. Similarly, two modified BEACOPP variants incorporating BV were compared in a

21 randomized phase II trial. $^{34}$ One of these variants, BrECADD (BV, etoposide,

22 cyclophosphamide, doxorubicin, dacarbazine, and dexamethasone) seems to be less toxic and

23 highly active and is currently compared to BEACOPPesc in the GHSG phase III HD21 trial

24 (NCT02661503). In addition, there are the promising immune checkpoint inhibitors which have

25 demonstrated impressive activity in Hodgkin lymphoma. ${ }^{35}$ These molecules have become 
1 crucial in the therapeutic armament of $\mathrm{HL}$ and currently are evaluated as part of first line

2 treatment (NCT03907488).

3 In conclusion, our study confirms that ABVD is an effective and well tolerated regimen

4 with similar survival rate as BEACOPPesc and supports its role as first line in advanced-stage

5 Hodgkin patients, irrespective of the IPS. However, the therapeutic horizon of Hodgkin

6 lymphoma is likely to change in the near future with a new generation of drugs that will

7 increasingly modify or even replace standard chemotherapies. This approach may eventually

8 supplant both ABVD and BEACOPPesc.

9

10

\section{Acknowledgements}

12 This article did not receive any specific grant from any funding agency in the public, commercial,

13 or not-for-profit sector

14

\section{Author Contributions}

16 PM designed and wrote the project, analyzed the data, reviewed the literature and wrote the 17 paper, CM, ID, JPB, FC, SF, BB, CC, DN, SDL, GM, CS, GL, SC collected the data, WD, WW, $18 \mathrm{MM}$ and DJS revised the paper, all the authors approved it.

19

20 Conflict of Interest

$21 \mathrm{PM}, \mathrm{CM}, \mathrm{ID}, \mathrm{J}-\mathrm{PB}, \mathrm{FC}, \mathrm{BB}, \mathrm{CC}, \mathrm{DN}, \mathrm{SDL}, \mathrm{GM}, \mathrm{WD}, \mathrm{GL}, \mathrm{SC}$ and DJS have no financial

22 disclosure. SF and MM have received honoraria from Mundipharma, WW has received

23 honoraria and research funding from Mundipharma and Roche. 
Mondello et al, 2020

\section{References}

2 1. Siegel RL, Miller KD, Jemal A. Cancer statistics, 2016. CA Cancer J Clin. 2015;66(1):n/a3 n/a. doi:10.3322/caac.21332

4 2. Glaser SL, Jarrett RF. The epidemiology of Hodgkin's disease. Baillieres Clin Haematol. 5 1996;9(3):401-416. doi:10.1016/s0950-3536(96)80018-7

6 3. Bonadonna G, Viviani S, Bonfante V, Gianni AM, Valagussa P. Survival in Hodgkin's 7 disease patients--report of 25 years of experience at the Milan Cancer Institute. Eur J $8 \quad$ Cancer. 2005;41(7):998-1006. doi:10.1016/j.ejca.2005.01.006

9 4. Evens AM, Hutchings M, Diehl V. Treatment of Hodgkin lymphoma: the past, present, 10

11 5. Longo DL, Glatstein E, Duffey PL, et al. Alternating MOPP and ABVD chemotherapy plus

6. Gobbi PG, Pieresca C, Federico M, et al. MOPP/EBV/CAD hybrid chemotherapy with or mantle-field radiation therapy in patients with massive mediastinal Hodgkin's disease. $J$ Clin Oncol. 1997;15(11):3338-3346. doi:10.1200/JCO.1997.15.11.3338 and future. Nat Clin Pract Oncol. 2008;5(9):543-556. doi:10.1038/ncponc1186

without limited radiotherapy in advanced or unfavorably presenting Hodgkin's disease: a report from the Italian Lymphoma Study Group. J Clin Oncol. 1993;11(4):712-719. doi:10.1200/JCO.1993.11.4.712

7. Gobbi PG, Pieresca C, Ghirardelli ML, et al. Long-term results from MOPPEBVCAD chemotherapy with optional limited radiotherapy in advanced Hodgkin's disease. Blood. 1998;91(8):2704-2712. http://www.ncbi.nIm.nih.gov/pubmed/9531579. Accessed October 11, 2019.

25 9. Viviani S, Zinzani PL, Rambaldi A, et al. ABVD versus BEACOPP for Hodgkin's 
Mondello et al, 2020

doi:10.1056/NEJMoa1100340

2 10. Merli F, Luminari S, Gobbi PG, et al. Long-Term Results of the HD2000 Trial Comparing ABVD Versus BEACOPP Versus COPP-EBV-CAD in Untreated Patients With Advanced Hodgkin Lymphoma: A Study by Fondazione Italiana Linfomi. J Clin Oncol. 2016;34(11):1175-1181. doi:10.1200/JCO.2015.62.4817

6 11. Federico M, Luminari S, lannitto E, et al. ABVD Compared With BEACOPP Compared 7

13. Mounier N, Brice P, Bologna S, et al. ABVD (8 cycles) versus BEACOPP (4 escalated cycles $\geq 4$ baseline): final results in stage III-IV low-risk Hodgkin lymphoma (IPS 0-2) of the LYSA H34 randomized trial†. Ann Oncol. 2014;25(8):1622-1628. doi:10.1093/annonc/mdu189

14. Swerdlow SH, Campo E, Pileri SA, et al. The 2016 revision of the World Health Organization classification of lymphoid neoplasms. Blood. 2016;127(20):2375-2390. doi:10.1182/blood-2016-01-643569

15. Cheson BD, Pfistner B, Juweid ME, et al. Revised response criteria for malignant lymphoma. J Clin Oncol. 2007;25(5):579-586. doi:10.1200/JCO.2006.09.2403

16. Cheson BD, Fisher RI, Barrington SF, et al. Recommendations for Initial Evaluation, Staging, and Response Assessment of Hodgkin and Non-Hodgkin Lymphoma: The Lugano Classification. J Clin Oncol. 2014;32(27):3059-3067. 
Mondello et al, 2020

$1 \quad$ doi:10.1200/JCO.2013.54.8800

2 17. Kumar A, Burger IA, Zhang Z, et al. Definition of bulky disease in early stage Hodgkin 3 lymphoma in computed tomography era: prognostic significance of measurements in the $4 \quad$ coronal and transverse planes. Haematologica. 2016;101(10):1237-1243.

5 doi:10.3324/haematol.2016.141846

6 18. Hasenclever D, Diehl V. A prognostic score for advanced Hodgkin's disease. N Engl J 7 Med. 1998;339(21):1506-1514. doi:10.1056/NEJM199811193392104

8 19. Federico M, Luminari S, lannitto E, et al. ABVD Compared With BEACOPP Compared 9 With CEC for the Initial Treatment of Patients With Advanced Hodgkin's Lymphoma:

10 Results From the HD2000 Gruppo Italiano per lo Studio dei Linfomi Trial. J Clin Oncol. $112009 ; 27(5): 805-811$. doi:10.1200/JCO.2008.17.0910

12 20. Borchmann P, Haverkamp H, Diehl V, et al. Eight cycles of escalated-dose BEACOPP 13 compared with four cycles of escalated-dose BEACOPP followed by four cycles of 14 baseline-dose BEACOPP with or without radiotherapy in patients with advanced-stage 15 hodgkin's lymphoma: final analysis of the HD12 trial of the German Hodgkin Study 16 Group. J Clin Oncol. 2011;29(32):4234-4242. doi:10.1200/JCO.2010.33.9549

17 21. Borchmann $\mathrm{P}$, Goergen $\mathrm{H}$, Kobe $\mathrm{C}$, et al. PET-guided treatment in patients with advanced-stage Hodgkin's lymphoma (HD18): final results of an open-label, international, randomised phase 3 trial by the German Hodgkin Study Group. Lancet (London, England). 2018;390(10114):2790-2802. doi:10.1016/S0140-6736(17)32134-7

22. Canellos GP, Anderson JR, Propert KJ, et al. Chemotherapy of Advanced Hodgkin's Disease with MOPP, ABVD, or MOPP Alternating with ABVD. N Engl J Med. 1992;327(21):1478-1484. doi:10.1056/NEJM199211193272102

24 23. Moccia AA, Donaldson J, Chhanabhai M, et al. International prognostic score in 25 advanced-stage Hodgkin's lymphoma: Altered utility in the modern era. $J$ Clin Oncol. 26 2012;30(27):3383-3388. doi:10.1200/JCO.2011.41.0910 
Mondello et al, 2020

124. Skoetz N, Trelle S, Rancea M, et al. Effect of initial treatment strategy on survival of patients with advanced-stage Hodgkin's lymphoma: A systematic review and network meta-analysis. Lancet Oncol. 2013;14(10):943-952. doi:10.1016/S1470-2045(13)70341-3

25. Johnson P, Federico M, Kirkwood A, et al. Adapted Treatment Guided by Interim PET-CT Scan in Advanced Hodgkin's Lymphoma. N Engl J Med. 2016;374(25):2419-2429. doi:10.1056/NEJMoa1510093

26. Press OW, Li H, Schöder H, et al. US Intergroup Trial of Response-Adapted Therapy for Stage III to IV Hodgkin Lymphoma Using Early Interim Fluorodeoxyglucose-Positron Emission Tomography Imaging: Southwest Oncology Group S0816. J Clin Oncol. 2016;34(17):2020-2027. doi:10.1200/JCO.2015.63.1119

27. Casasnovas O, Brice P, Bouabdallah R, et al. Randomized Phase III Study Comparing an Early PET Driven Treatment De-Escalation to a Not PET-Monitored Strategy in Patients with Advanced Stages Hodgkin Lymphoma: Interim Analysis of the AHL2011 Lysa Study. Blood. 2015;126(23). http://www.bloodjournal.org/content/126/23/577?ssochecked=true. Accessed November 9, 2018.

28. Engert A, Haverkamp H, Kobe $\mathrm{C}$, et al. Reduced-intensity chemotherapy and PET-guided radiotherapy in patients with advanced stage Hodgkin's lymphoma (HD15 trial): a randomised, open-label, phase 3 non-inferiority trial. Lancet. 2012;379(9828):1791-1799. doi:10.1016/S0140-6736(11)61940-5

29. Bari A, Marcheselli R, Sacchi S, et al. The classic prognostic factors in advanced Hodgkin's lymphoma patients are losing their meaning at the time of Pet-guided treatments. Ann Hematol. 2020;99(2):277-282. doi:10.1007/s00277-019-03893-7

30. Gallamini A, Tarella C, Viviani S, et al. Early Chemotherapy Intensification With Escalated BEACOPP in Patients With Advanced-Stage Hodgkin Lymphoma With a Positive Interim Positron Emission Tomography/Computed Tomography Scan After Two ABVD Cycles: Long-Term Results of the GITIL/FIL HD 0607 Trial. J Clin Oncol. 2018;36(5):454-462. 
Mondello et al, 2020

1

2 31. Stephens DM, Li H, Schöder H, et al. Five-year follow-up of SWOG S0816: limitations 3

4

5 32. Younes A, Gopal AK, Smith SE, et al. Results of a Pivotal Phase II Study of Brentuximab 6 7

11 34. Eichenauer DA, Plütschow A, KreissI S, et al. Incorporation of brentuximab vedotin into 12 Vedotin for Patients With Relapsed or Refractory Hodgkin's Lymphoma. J Clin Oncol. 2012;30(18):2183-2189. doi:10.1200/JCO.2011.38.0410

33. Connors JM, Jurczak W, Straus DJ, et al. Brentuximab Vedotin with Chemotherapy for Stage III or IV Hodgkin's Lymphoma. N Engl J Med. 2018;378(4):331-344. doi:10.1056/NEJMoa1708984 first-line treatment of advanced classical Hodgkin's lymphoma: final analysis of a phase 2 randomised trial by the German Hodgkin Study Group. Lancet Oncol. 2017;18(12):16801687. doi:10.1016/S1470-2045(17)30696-4

35. Ansell SM, Lesokhin AM, Borrello I, et al. PD-1 blockade with nivolumab in relapsed or refractory Hodgkin's lymphoma. N Engl J Med. 2015;372(4):311-319. doi:10.1056/NEJMoa1411087 
Mondello et al, 2020

\section{Figure Legends}

2 Figure 1. Severe hematologic toxicities. Bar graph showing the frequency of acute grade 3-4

3 hematologic adverse events that occurred in advanced-stage Hodgkin's lymphoma patients

4 treated with either BEACOPPesc (black) or ABVD (gray). Differences between groups were

5 calculated with the Student $t$ test. ${ }^{* *} p=0.002 ;{ }^{* * *} p<0.001$.

6

7 Figure 2. Kaplan-Meier analysis of progression-free survival $(\mathbf{A} ; p=0.11)$ and overall survival $(\mathbf{B}$;

$8 p=0.94)$ for advanced-stage Hodgkin's lymphoma patients treated with either BEACOPP

9 escalated (black) or ABVD (gray). BEACOPP, bleomycin, etoposide, doxorubicin,

10 cyclophosphamide, vincristine, procarbazine and prednisone; ABVD, doxorubicin, bleomycin,

11 vinblastine and dacarbazine.

\section{Supplemental Figure Legends}

15 Supplemental Figure 1. Kaplan-Meier analysis of progression-free survival and overall survival 16 in PET2-negative (A-C) and PET2-positive (B-D) advanced-stage Hodgkin's lymphoma patients 17 treated with either BEACOPP escalated (black) or ABVD (gray). BEACOPP, bleomycin,

18 etoposide, doxorubicin, cyclophosphamide, vincristine, procarbazine and prednisone; ABVD,

19 doxorubicin, bleomycin, vinblastine and dacarbazine.

21 Supplemental Figure 2. Kaplan-Meier analysis of progression-free survival and overall survival 22 in advanced-stage Hodgkin's lymphoma patients with International Prognostic Score $<3$ (A-C) 23 and $\geq 3$ (B-D) who were treated with either BEACOPP escalated or ABVD. BEACOPP, 24 bleomycin, etoposide, doxorubicin, cyclophosphamide, vincristine, procarbazine and 25 prednisone; ABVD, doxorubicin, bleomycin, vinblastine and dacarbazine; IPS, International 26 Prognostic Score. 
Table 1. Baseline patient characteristics

\begin{tabular}{|c|c|c|c|c|c|}
\hline & $\begin{array}{r}\text { BEACO } \\
(n=1\end{array}$ & $\begin{array}{l}\text { Pesc } \\
\text { 1) }\end{array}$ & & & p-value \\
\hline & $\mathrm{n}$ & $\%$ & $\mathrm{n}$ & $\%$ & \\
\hline $\begin{array}{l}\text { Median, years } \\
>50 \text { years }(\text { EORTC) } \\
>60 \text { years }\end{array}$ & $\begin{array}{c}37 \\
24 \\
4\end{array}$ & $\begin{array}{l}\text { N.A. } \\
19.8 \\
3.3\end{array}$ & $\begin{array}{c}36.5 \\
60 \\
11\end{array}$ & $\begin{array}{l}\text { N.A. } \\
21.7 \\
4\end{array}$ & $\begin{array}{l}\text { N.A } \\
0.42 \\
0.77\end{array}$ \\
\hline $\begin{array}{l}\text { Female } \\
\text { Male }\end{array}$ & $\begin{array}{l}29 \\
92\end{array}$ & $\begin{array}{l}24 \\
76\end{array}$ & $\begin{array}{c}99 \\
177\end{array}$ & $\begin{array}{l}35.8 \\
64.1\end{array}$ & $\begin{array}{l}0.06 \\
0.08\end{array}$ \\
\hline $\begin{array}{l}\text { Hodgkin subtype } \\
\text { Nodular sclerosing } \\
\text { Mixed cellularity } \\
\text { Lymphocytes depleted } \\
\text { Lymphocytes rich }\end{array}$ & $\begin{array}{c}91 \\
20 \\
9 \\
1\end{array}$ & $\begin{array}{c}75.2 \\
16.5 \\
7.4 \\
0.9\end{array}$ & $\begin{array}{c}211 \\
42 \\
21 \\
2\end{array}$ & $\begin{array}{l}76.4 \\
15.2 \\
7.6 \\
0.8\end{array}$ & $\begin{array}{l}0.77 \\
0.54 \\
0.82 \\
0.88\end{array}$ \\
\hline B-Symptoms & 72 & 59.5 & 147 & 53.2 & 0.23 \\
\hline Bone marrow involvement & 20 & 16.5 & 25 & 9.0 & 0.08 \\
\hline Bulky disease (> $7 \mathrm{~cm})$ & 25 & 20.6 & 61 & 22.1 & 0.11 \\
\hline Mediastinal mass & 18 & 14.8 & 48 & 17.4 & 0.37 \\
\hline Elevated LDH & 44 & 36.4 & 97 & 35.1 & 0.83 \\
\hline Elevated B2M & 31 & 25.6 & 60 & 21.7 & 0.28 \\
\hline Elevated ESR & 70 & 57.8 & 156 & 56.5 & 0.77 \\
\hline $\mathrm{Hb}<10.5 \mathrm{~g} / \mathrm{dL}$ & 28 & 23.1 & 55 & 19.9 & 0.09 \\
\hline$W B C>15,000 / \mathrm{mm} 3$ & 25 & 20.6 & 64 & 23.2 & 0.43 \\
\hline Lymphocytes $<600 / \mathrm{mm} 3$ & 15 & 12.4 & 33 & 11.9 & 0.75 \\
\hline Monocytes >900/mm3 & 13 & 10.7 & 31 & 11.2 & 0.41 \\
\hline PLT $<150,000$ & 8 & 6.6 & 11 & 4.0 & 0.09 \\
\hline Albumin $<4 \mathrm{~g} / \mathrm{dL}$ & 28 & 23.1 & 57 & 20.6 & 0.18 \\
\hline $\begin{array}{l}\text { Stage } \\
\text { III } \\
\text { IV }\end{array}$ & $\begin{array}{l}59 \\
62\end{array}$ & $\begin{array}{l}48.8 \\
51.2\end{array}$ & $\begin{array}{l}139 \\
137\end{array}$ & $\begin{array}{l}50.3 \\
49.7\end{array}$ & $\begin{array}{l}0.11 \\
0.15\end{array}$ \\
\hline $\begin{array}{c}\text { ECOG } \\
\begin{array}{c}0-1 \\
>1\end{array}\end{array}$ & $\begin{array}{l}99 \\
22\end{array}$ & $\begin{array}{l}81.8 \\
18.2\end{array}$ & $\begin{array}{l}198 \\
78\end{array}$ & $\begin{array}{l}71.7 \\
28.2\end{array}$ & $\begin{array}{l}0.062 \\
0.065\end{array}$ \\
\hline $\begin{array}{l}\text { International Prognostic } \\
\text { Score (IPS) } \\
0-1 \\
2-3 \\
4-7\end{array}$ & $\begin{array}{l}15 \\
52 \\
54\end{array}$ & $\begin{array}{l}12.4 \\
43 \\
44.6\end{array}$ & $\begin{array}{c}78 \\
103 \\
95\end{array}$ & $\begin{array}{l}28.3 \\
37.3 \\
34.4\end{array}$ & $\begin{array}{l}0.041 \\
0.071 \\
0.065\end{array}$ \\
\hline
\end{tabular}

Abbreviations: ABVD, doxorubicin, bleomycin, vinblastine and dacarbazine; BEACOPPesc, escalated bleomycin, etoposide, doxorubicin, cyclophosphamide, vincristine, procarbazine and prednisone; LDH, lactate dehydrogenase; B2M, Beta2 microglobulin; ESR, erythrocyte sedimentation rate; $\mathrm{Hb}$, hemoglobin; WBC, white blood count; PLT, platelet. 
Figure 1

10

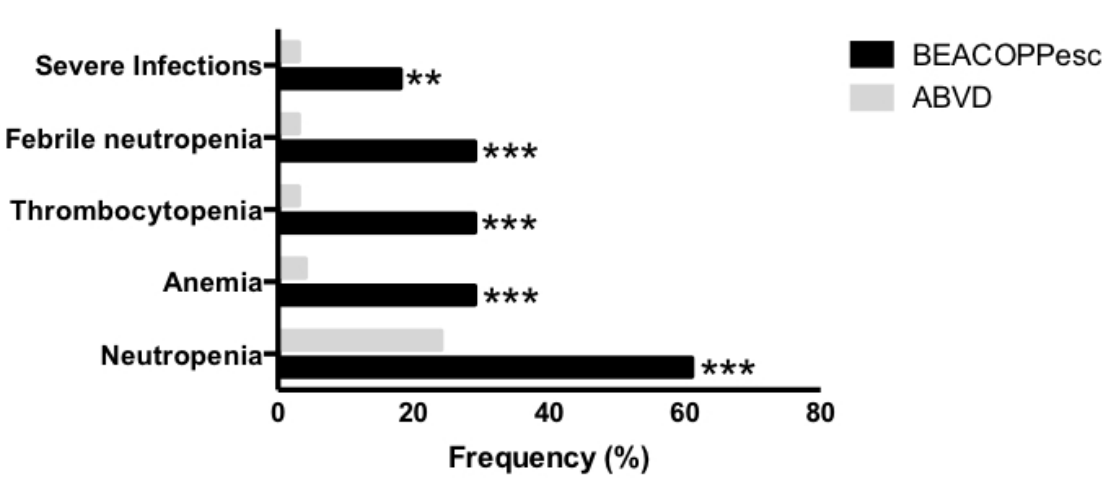

Figure 1. Severe hematologic toxicities. Bar graph showing the frequency of acute grade 3-4 hematologic adverse events that occurred in advanced-stage Hodgkin's lymphoma patients treated with either BEACOPPesc (black) or ABVD (gray). Differences between groups were calculated with the Student $\mathrm{t}$ test. ** $\mathrm{p}=0.002 ; * * * \mathrm{p}<0.001$.

$190 \times 254 \mathrm{~mm}(96 \times 96 \mathrm{DPI})$ 


\section{Figure 2}

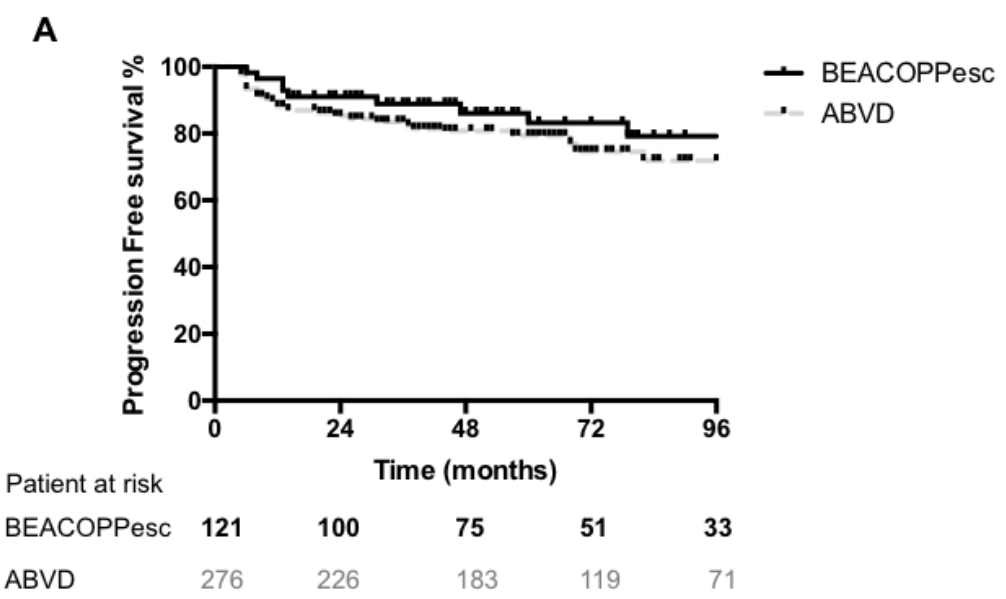

B

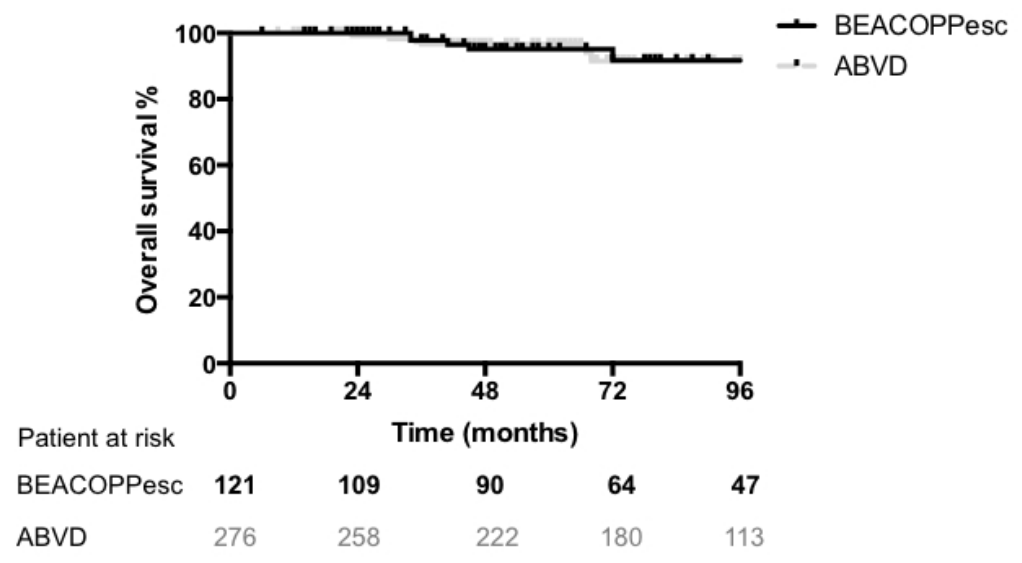

Figure 2. Kaplan-Meier analysis of progression-free survival $(A ; p=0.11)$ and overall survival $(B ; p=0.94)$ for advanced-stage Hodgkin's lymphoma patients treated with either BEACOPP escalated (black) or ABVD (gray). BEACOPP, bleomycin, etoposide, doxorubicin, cyclophosphamide, vincristine, procarbazine and prednisone; ABVD, doxorubicin, bleomycin, vinblastine and dacarbazine.

$$
190 \times 254 \mathrm{~mm}(96 \times 96 \mathrm{DPI})
$$


Table 2. Secondary Malignancies by treatment group.

\begin{tabular}{|lcc|}
\hline Secondary Malignancy & $\begin{array}{c}\text { BEACOPPesc } \\
(\mathbf{n = 1 2 1 )}\end{array}$ & $\begin{array}{c}\text { ABVD } \\
(\mathbf{n = 2 7 6 )}\end{array}$ \\
\hline Lung cancer & 1 & 1 \\
\hline Breast cancer & 4 & 1 \\
\hline Thyroid cancer & 1 & - \\
\hline Melanoma & 1 & - \\
\hline Myelodysplasia & 3 & - \\
\hline Acute leukemia & 1 & \\
\hline
\end{tabular}

Abbreviations: BEACOPPesc, escalated bleomycin, etoposide, doxorubicin, cyclophosphamide, vincristine, procarbazine, and prednisone; ABVD, doxorubicin, bleomycin, vinblastine, and dacarbazine. 